COMUNICAÇÃO CIENTÍFICA

\title{
Primeiro registro de Myotis riparius Handley (Mammalia, Chiroptera, Vespertilionidae) no Estado do Rio de Janeiro, sudeste do Brasil
}

\author{
Daniela Dias \& Adriano Lúcio Peracchi
}

Laboratório de Mastozoologia, Instituto de Biologia, Universidade Federal Rural do Rio de Janeiro. Antiga Rodovia Rio-São Paulo, km 47, 23890-000 Seropédica, Rio de Janeiro, Brasil. Bolsista do CNPq.

E-mail: dani_dias262@yahoo.com.br; aperacchi@webdigital.com.br

\begin{abstract}
First record of Myotis riparius Handley (Mammalia, Chiroptera, Vespertilionidae) in the Rio de Janeiro State, southeastern Brazil. In this paper, the first occurrence of the vespertilionid bat Myotis riparius Handley, 1960 in the Rio de Janeiro State, southeastern Brazil is reported. Seven specimens were caught with mist nets in the Reserva Biologica do Tinguá, an Atlantic Forest area in the Nova Iguaçu County, Rio de Janeiro state. Taxonomic aspects of this species are discussed. Measurements obtained for the specimens are also provided. KEY WORDS. Atlantic Forest; bats; distribution; taxonomy.
\end{abstract}

RESUMO. No presente trabalho, é registrada a primeira ocorrência do morcego vespertilionídeo Myotis riparius Handley, 1960 no Estado do Rio de Janeiro. Sete exemplares dessa espécie foram capturados com redes de espera ("mist nets") na Reserva Biológica do Tinguá, uma área de Mata Atlântica no Município de Nova Iguaçu, Estado do Rio de Janeiro. Aspectos taxonômicos dessa espécie são discutidos e medidas obtidas para os exemplares são fornecidas.

PALAVRAS-CHAVE. Distribuição; Mata Atlântica; morcegos; taxonomia.

A Reserva Biológica do Tinguá $\left(22^{\circ} 28^{\prime}-22^{\circ} 39^{\prime} \mathrm{S}, 43^{\circ} 13^{\prime}-\right.$ $43^{\circ} 34^{\prime} \mathrm{W}$ ), com 26.000 ha, $150 \mathrm{~km}^{2}$ de área e distribuída entre os municípios de Nova Iguaçu, Duque de Caxias, Petrópolis e Miguel Pereira, constitui um dos últimos trechos conservados de Mata Atlântica no Estado do Rio de Janeiro, sendo o único remanescente com áreas íntegras na região da Baixada Fluminense (Lima 2002). Durante inventário da quiropterofauna conduzido na Reserva, foram colecionados sete exemplares adultos de Myotis riparius Handley, 1960 com epífises dos metacarpos e falanges totalmente ossificadas. Todos os exemplares foram incorporados à Coleção Adriano Lúcio Peracchi (ALP), depositada no Laboratório de Mastozoologia, Instituto de Biologia da Universidade Federal Rural do Rio de Janeiro (UFRRJ).

O primeiro exemplar (ALP 6247), uma fêmea que não apresentava indícios de atividade reprodutiva, foi capturado em 13/III/2001, com rede armada ao nível do solo na Estrada do Macuco, próximo à represa da CEDAE. Em 14/VIII/2004, foram obtidos um macho (ALP 6622) e uma fêmea (ALP 6623) inativos, com rede ao nível do solo perto de uma captação de água da CEDAE, em Serra Velha. Em 15/I/2005, foi colecionada uma fêmea inativa (ALP 6625), com rede ao nível do solo armada em uma trilha situada próximo ao Rio Doce, na locali- dade conhecida como Sítio Rio Doce, na Estrada da Janjana, cerca de $820 \mathrm{~m}$ de altitude. Os outros três exemplares de $M$. riparius foram capturados também no Sítio Rio Doce, em 25/ III/2006, com rede armada ao nível do solo, na entrada de outra trilha que dá acesso a uma piscina natural formada por uma pequena cachoeira. Nenhum dos exemplares, um macho (ALP 6680) e duas fêmeas (ALP 6681 e ALP 6682), apresentava indícios de atividade reprodutiva. Medidas (em $\mathrm{mm}$ ) obtidas para os exemplares, conforme critérios propostos por LóPEz-GonZÁLEZ et al. (2001), são apresentadas na tabela I.

Descrita por Handley (1960) como subespécie de M. simus (Thomas, 1901) com base em exemplares colecionados no Panamá, $M$. riparius foi posteriormente reconhecida como espécie válida por LAVAL (1973). Tal arranjo tem sido seguido por autores subseqüentes (BAUd \& Menu 1993, Simmons \& Voss 1998, BARquez et al. 1999, López-GonZÁlez et al. 2001, Simmons 2005, Peracchi et al. 2006).

Além de M. riparius, outras cinco espécies de Myotis Kaup, 1829 são reconhecidas para o Brasil (Simmons 2005, Peracchi et al. 2006): M. albescens (E. Geoffroy, 1806), M. levis (I. Geoffroy, 1824), M. nigricans (Schinz, 1821), M. ruber (E. Geoffroy, 1806) e M. simus (Thomas, 1901). Medidas e caracteres taxonômicos para estas espécies são fornecidos por LAVAL (1973), BAUD \& 
Menu (1993), Simmons \& Voss (1998), BARQuez et al. (1999) e LópezGonZález et al. (2001). Para distinguir M. riparius de M. nigricans, LAVAL (1973) e López-GonZÁlez et al. (2001) apontam pelagem mais curta e lanosa, segundo pré-molar superior deslocado para o lado lingual, presença de crista sagital e proporção largura entre caninos/ largura pós-orbitária (Lc/Lpo) superior a 1, como caracteres mais importantes.

Os sete exemplares colecionados na Reserva Biológica do Tinguá foram identificados como $M$. riparius de acordo com os caracteres diagnósticos fornecidos pelos autores acima citados. Contudo, o caráter "segundo pré-molar superior deslocado para o lado lingual", considerado pelos autores como importante para distinguir M. riparius de M. nigricans, foi observado em apenas um exemplar (fêmea ALP 6623). Em sua revisão de Myotis do Paraguai, López-GonzÁLez et al. (2001) encontraram grande variação quanto à posição do segundo pré-molar superior; nos 59 exemplares de $M$. riparius examinados pelos autores, somente 16 possuíam o segundo pré-molar superior deslocado para o lado lingual. Além disso, os autores também observaram que alguns exemplares de M. nigricans no Paraguai possuíam o segundo prémolar superior deslocado para o lado lingual e, no Paraguai, a pelagem de $M$. riparius não era tão distintivamente curta e lanosa quanto no norte da América do Sul. Tais variações podem tornar difícil a identificação correta. López-GonzÁLEz et al. (2001) recomendam utilizar a presença de crista sagital e a proporção Lc/Lpo > 1 como caracteres mais seguros para separar M. riparius de $M$. nigricans. De fato, todos os exemplares de $M$. riparius da Reserva Biológica do Tinguá apresentam crista sagital e Lc/Lpo >
1; nenhum dos cinco exemplares de $M$. nigricans colecionados na Reserva possui crista sagital e para todos estes a proporção Lc/Lpo é menor que um (Tab. I).

Apesar de não se apresentar deslocado para o lado lingual (exceto em um exemplar), o segundo pré-molar superior nos exemplares de $M$. riparius examinados é mais reduzido que o primeiro em vista oclusal e se apresenta um pouco mais comprimido entre o primeiro e o terceiro pré-molares superiores. Nos exemplares de $M$. nigricans, o segundo pré-molar superior é praticamente subigual ao primeiro em vista oclusal e não é comprimido entre o primeiro e o terceiro.

Com relação à textura da pelagem, apontada como caráter útil para separar as duas espécies, não obstante a variação encontrada por LóPEz-GonZÁlez et al. (2001), foi possível observar no campo e em quatro exemplares de $M$. riparius preservados sob a forma de pele cheia (machos ALP 6622 e ALP 6680, fêmeas ALP 6623 e ALP 6681) a pelagem curta e lanosa, em contraste com os exemplares de M. nigricans (pelagem mais longa e lisa).

Simmons \& Voss (1998) encontraram variação quanto à pelagem (coloração, textura e bandeamento) em $M$. riparius e M. nigricans em Paracou (Guiana Francesa) e relataram que a identificação segura só foi possível com os crânios limpos e com base nas medidas cranianas; os autores constataram que os exemplares de $M$. riparius eram maiores que os de $M$. nigricans em comprimento total do crânio, comprimento côndilo-incisivo, série de dentes superiores, largura zigomática e largura entre os molares. Na Reserva Biológica do Tinguá, as medidas dos machos de $M$. riparius são maiores que as dos machos de $M$.

Tabela I. Medidas de exemplares de Myotis nigricans e Myotis riparius colecionados na Reserva Biológica do Tinguá, Estado do Rio de Janeiro, sudeste do Brasil. (N) Número de exemplares, (Min) mínimo, (Max) máximo, (Lc/Lpo) largura entre os caninos, dividida pela largura pós-orbitária.

\begin{tabular}{|c|c|c|c|c|}
\hline \multirow{3}{*}{ Medidas } & \multicolumn{2}{|c|}{ Macho } & \multicolumn{2}{|c|}{ Fêmea } \\
\hline & M. nigricans $(\mathrm{N}=2)$ & M. riparius $(\mathrm{N}=2)$ & M. nigricans $(\mathrm{N}=3)$ & M. riparius $(\mathrm{N}=5)$ \\
\hline & Min - Máx & Min - Máx & Min - Máx & Min - Máx \\
\hline Antebraço & $31,38-33,74$ & $35,04-35,14$ & $33,10-33,64$ & $34,10-35,16$ \\
\hline Comprimento total do crânio & $13,52-13,64$ & $13,78-13,98$ & $13,64-13,82$ & $13,54-13,88$ \\
\hline Comprimento côndilo-incisivo & $12,66-12,96$ & $13,22-13,22$ & $12,94-13,24$ & $12,90-13,14$ \\
\hline Comprimento basal & $11,14-11,56$ & $11,50-11,54$ & $11,42-11,76$ & $11,36-12,50$ \\
\hline Comprimento palatal & $6,76-6,82$ & $6,56-7,14$ & $6,24-6,64$ & $6,70-7,00$ \\
\hline Série de dentes superiores & $4,98-5,20$ & $5,28-5,30$ & $4,94-5,10$ & $5,14-5,28$ \\
\hline Largura entre os molares & $5,18-5,24$ & $5,38-5,42$ & $5,12-5,26$ & $5,10-5,68$ \\
\hline Largura entre os caninos & $3,32-3,38$ & $3,72-3,76$ & $3,32-3,44$ & $3,48-3,92$ \\
\hline Largura pós-orbitária & $3,46-3,62$ & $3,66-3,66$ & $3,64-3,68$ & $3,48-3,58$ \\
\hline Largura zigomática & $8,00-8,14$ & $8,44-8,76$ & $7,10-8,18$ & $7,90-8,72$ \\
\hline Largura da caixa craniana & $6,38-6,40$ & $6,54-6,70$ & $6,56-6,82$ & $6,46-6,96$ \\
\hline Largura mastóide & $6,70-6,96$ & $7,00-7,14$ & $6,68-7,06$ & $7,02-7,42$ \\
\hline Comprimento da mandíbula & $9,26-9,34$ & $9,66-9,78$ & $9,40-9,66$ & $9,72-9,98$ \\
\hline Série de dentes inferiores & $5,54-5,66$ & $5,76-5,94$ & $5,10-5,74$ & $5,66-6,00$ \\
\hline Lc/Lpo & $0,92-0,96$ & $1,02-1,03$ & $0,83-0,93$ & $1,00-1,09$ \\
\hline
\end{tabular}


nigricans; no caso das fêmeas, apesar da sobreposição com $M$. nigricans em alguns caracteres (devido às pequenas dimensões do exemplar ALP 6247), as medidas de M. riparius são em geral também maiores (Tab. I).

Myotis riparius distribui-se de Honduras até o Uruguai, passando pelo Brasil, Argentina, Paraguai e Bolívia e Trinidad (Simmons 2005). No território brasileiro, essa espécie tem sido registrada na Amazônia (Voss \& Emmons 1996, Nogueira et al. 1999, Bernard 2001, 2002, Bernard \& Fenton 2002); nos estados da Bahia, nordeste brasileiro (FARIA et al. 2006), Mato Grosso, centro-oeste, em área de Cerrado (Gonçalves \& Gregorin 2004), Paraná (SEKiAMa et al. 2001, MiretZKi 2003, BiAnConi et al. 2004), Santa Catarina (Cherem et al. 2004, Graciolli 2004) e Rio Grande do Sul (González \& Fabí́n 1995), sul do Brasil. No sudeste brasileiro, Bertola et al. (2005) registrou um exemplar colecionado no Parque Estadual da Cantareira, Estado de São Paulo e LAVAL (1973) examinou um exemplar colecionado em Viçosa, Minas Gerais. MARINHo-Filho (1996) mencionou a ocorrência de $M$. riparius no Rio de Janeiro, mas não forneceu dados sobre localidade exata nem número de registro em coleção de qualquer exemplar. Essa espécie não foi listada para o Estado por EsberáRd \& Bergallo (2005). A série de M. riparius obtida na Reserva Biológica do Tinguá constitui, portanto, o primeiro registro comprovado dessa espécie para o estado do Rio de Janeiro.

\section{AGRADECIMENTOS}

Ao IBAMA, pelas Licenças de Pesquisa em Unidade de Conservação concedidas (Licenças nº 027/2000, 042/2004 e 239/2005); à Chefia da Reserva Biológica do Tinguá (Maria L. Xavier e Luís H. Teixeira) pela oportunidade e apoio para realizar trabalhos de campo na Reserva; a Valter dos Santos, Paulo C. de Oliveira Jr, Dayana P. Bolzan e Mayara A. Martins pelo apoio e auxílio nos trabalhos de campo; ao CNPq pela Bolsa de Doutorado concedida à D. Dias; ao CNPq e FAPERJ pelas Bolsas concedidas a A.L. Peracchi; aos servidores do Instituto de Biologia, UFRRJ, pelo auxílio.

\section{REFERÊNCIAS BIBLIOGRÁFICAS}

Barquez, R.M.; M.A. Mares \& J.K. Braun. 1999. The bats of Argentine. Special Publications of the Museum, Texas Tech University 42: 1-275.

Baud, F.J. \& H. Menu. 1993. Paraguayan bats of the genus Myotis, with a redefinition of M. simus (Thomas, 1901). Revue Suisse de Zoologie 100 (3): 595-607.

Bernard, E. 2001. Vertical stratification of bat communities in primary forests of Central Amazônia, Brazil. Journal of Tropical Ecology 17: 115-126.

BERNARD, E. 2002. Diet, activity and reproduction of bat species (Mammalia, Chiroptera) in Central Amazonia, Brazil. Revista Brasileira de Zoologia 19 (1): 173-188.

Bernard, E. \& M.B. Fenton. 2002. Species diversity of bats (Mammalia: Chiroptera) in forest fragments, primary forests, and savannas in central Amazonia, Brazil. Canadian Journal of Zoology 80: 1124-1140.

Bertola, P.B.; C.C. Aires; S.E. Favorito; G. Graciolli; M. Amaku \& R. Pinto-DA-Rocha. 2005. Bat flies (Díptera: Streblidae, Nycteribiidae) parasitic on bats (Mammalia: Chiroptera) at Parque Estadual da Cantareira, São Paulo, Brazil: parasitism rates and host-parasite associations. Memórias do Instituto Oswaldo Cruz 100 (1): 25-32.

Bianconi, G.V.; S.B. Mikich \& W.A. Pedro. 2004. Diversidade de morcegos (Mammalia, Chiroptera) em remanescentes florestais do município de Fênix, noroeste do Paraná, Brasil. Revista Brasileira de Zoologia 21 (4): 943-954.

Cherem, J.J.; P.C. Simões-Lopes; S.A. Althoff \& M.E. Graipel. 2004. Lista dos mamíferos do Estado de Santa Catarina, Sul do Brasil. Mastozoología Neotropical 11 (2): 151-184.

Esberárd, C.E.L. \& H.G. Bergallo. 2005. Research on bats in the state of Rio de Janeiro, Southeastern Brazil. Mastozoologia Neotropical 12 (2): 237-243.

Faria, D.; B. Soares-Santos \& E. SAmpaio. 2006. Bats from the Atlantic rainforest of southern Bahia, Brazil. Biota Neotropica 6 (2): 1-13.

Gonçalves, E. \& R. Gregorin. 2004. Quirópteros da Estação Ecológica da Serra das Araras, Mato Grosso, Brasil, com o primeiro registro de Artibeus gnomus e A. anderseni para o cerrado. Lundiana 5 (2): 143-149.

GonzÁlez, J.C. \& M.E. Fabián. 1995. Una nueva especie de murcielago para el Estado de Rio Grande do Sul, Brasil: Myotis riparius Handley, 1960 (Chiroptera: Vespertilionidae). Comunicações do Museu de Ciência e Tecnologia, PUCRS, Série Zoologia 8: 55-59.

Graciolli, G. 2004. Nycteribiidae (Díptera, Hippoboscoidea) no Sul do Brasil. Revista Brasileira de Zoologia 21 (4): 971985.

Handley JR, C.O. 1960. Descriptions of new bats from Panama. Proceedings of the United States National Museum, Smithsonian Institution 112 (3442): 459-479.

LAVAL, R.K. 1973. A revision of the neotropical bats of the genus Myotis. Natural History Museum, Los Angeles County, Science Bulletin 15: 1-54.

Lima, H.C. 2002. Paisagem e flora da Reserva Biológica do Tinguá: subsídios ao monitoramento da vegetação. Rio de Janeiro, Instituto de Pesquisas Jardim Botânico do Rio de Janeiro, 45p.

López-Gonzalez, C.; S.J. Presley; R.D. Owen \& M.R. Willig. 2001. Taxonomic status of Myotis (Chiroptera: Vespertilionidae) in Paraguay. Journal of Mammalogy 82 (1): 138-160.

Marinho-Filho, J. 1996. Distribution of bat diversity in the southern and southeastern Brazilian Atlantic Forest. Chiroptera Neotropical 2 (2): 51-54.

Miretzki, M. 2003. Morcegos do Estado do Paraná, Brasil (Mammalia, Chiroptera): riqueza de espécies, distribuição e síntese do conhecimento atual. Papéis Avulsos de Zoologia 43 (6): 101-138. 
Nogueira, M.R.; A. Pol \& A.L. Peracchi. 1999. New records of bats from Brazil with a list of additional species for the chiropteran fauna of the state of Acre, western Amazon basin. Mammalia 63 (3): 363-368.

Peracchi, A.L.; I.P. Lima; N.R. Reis; M.R. Nogueira \& H. Ortencio Filho. 2006. Ordem Chiroptera, p. 153-230. In: N.R. ReIs; A.L. Peracchi; W.A. Pedro \& I.P. Lima (Eds). Mamíferos do Brasil. Londrina, Editora da Universidade Estadual de Londrina, 437p.

Sekiama, M.L.; N.R. Reis; A.L. Peracchi \& V.J. Rocha. 2001. Morcegos do Parque Nacional do Iguaçu, Paraná (Chiroptera, Mammalia). Revista Brasileira de Zoologia 18 (3): 749-754.
Simmons, N.B. 2005. Order Chiroptera, p. 312-529. In: D.E. WILSON \& D.M. REEDER (Eds). Mammal species of the world: a taxonomic and geografic reference. Baltimore, The Johns Hopkins University Press, $3^{\text {rd }}$ ed., XXXVIII+2142p.

Simmons, N.B. \& R.S. Voss. 1998. The mammals of Paracou, French Guiana: a neotropical lowland rainforest fauna. Part I. Bats. Bulletin of the American Museum of Natural History 273: 1-219.

Voss, R.S. \& L.H. Emmons. 1996. Mammalian diversity in Neotropical lowland rainforests: a preliminary assessment. Bulletin of the American Museum of Natural History 230: 1-115.

Recebido em 04.X.2006; aceito em 15.V.2007. 